STRUCTURAL BIOLOGY COMMUNICATIONS

ISSN 2053-230X

\section{Re-refinement of Plasmodium falciparum orotidine 5'-monophosphate decarboxylase provides a clearer picture of an important malarial drug target}

\author{
Walter R. P. Novak, ${ }^{a}$ Korbin H. J. West, ${ }^{a}$ Lucy M. D. Kirkman ${ }^{b}$ and \\ Gabriel S. Brandt ${ }^{\mathbf{b}_{*}}$
}

Received 13 June 2018

Accepted 23 July 2018

Edited by S. Sheriff, Bristol-Myers Squibb, USA

Keywords: orotidine 5'-monophosphate decarboxylase; Plasmodium falciparum; Michaelis complex; re-refinement.

PDB references: orotidine 5'-monophosphate decarboxylase, 6dsq; 6dsr; 6dss

Supporting information: this article has supporting information at journals.iucr.org/f

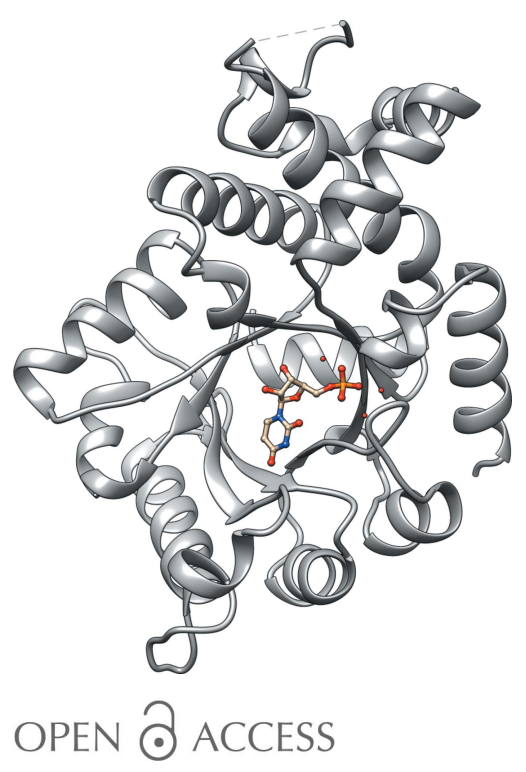

${ }^{a}$ Chemistry Department, Wabash College, 301 West Wabash Avenue, Crawfordsville, IN 47933, USA, and ${ }^{\mathbf{b} C h e m i s t r y}$ Department, Franklin and Marshall College, PO Box 3003, Lancaster, PA 17604, USA. *Correspondence e-mail: gbrandt@fandm.edu

The development of antimalarial drugs remains a public health priority, and the orotidine 5'-monophosphate decarboxylase from Plasmodium falciparum (PfOMPDC) has great potential as a drug target. The crystallization of PfOMPDC with substrate bound represents an important advance for structurebased drug-design efforts [Tokuoka et al. (2008), J. Biochem. 143, 69-78]. The complex of the enzyme bound to the substrate OMP (PDB entry 2za1) would be of particular utility in this regard. However, re-refinement of this structure of the Michaelis complex shows that the bound ligand is the product rather than the substrate. Here, the re-refinement of a set of three structures, the apo enzyme and two versions of the product-bound form (PDB entries 2za1, 2za2 and 2za3), is reported. The improved geometry and fit of these structures to the observed electron density will enhance their utility in antimalarial drug design.

\section{Introduction}

Protein structures, particularly those with substrates bound, have been invaluable in helping to illuminate the details of enzyme mechanisms and the design of novel inhibitors (Petsko \& Ringe, 2004). In order to provide insight into the workings of enzymes, protein structures must be interpreted in the light of, and are limited by, their ability to capture atomiclevel detail (Wlodawer et al., 2018). The enzyme orotidine 5 -monophosphate decarboxylase (OMPDC) is involved in the synthesis of pyrimidine nucleotides and is essential in malaria parasites (Rathod \& Reyes, 1983). Unlike their human hosts, who have a salvage pathway, malaria parasites rely entirely on de novo nucleotide synthesis, so OMPDC has been proposed as a potential therapeutic target (Cassera et al., 2011). In addition, the ability of OMPDC to efficiently catalyze decarboxylation without a cofactor has attracted the interest of mechanistic enzymologists (Richard et al., 2018; Lewis et al., 2017; Reyes et al., 2015; Vardi-Kilshtain et al., 2013; Lee \& Houk, 1997). For both of these reasons, the structure of OMPDC from Plasmodium falciparum (PfOMPDC) with the substrate orotidine $5^{\prime}$-monophosphate (OMP) bound would be of considerable scientific interest. The series of structures with PDB codes 2za2, 2za1 and 2za3 represents an attempt to capture the enzyme alone, with the OMP substrate and with its product uridine 5'-monophosphate (UMP), respectively (Tokuoka et al., 2008).

Inspection of these models reveals significant imperfections in terms of backbone geometry, density fit and ligand selection (Table 1). The $P D B-R E D O$ server is often able to make 
Table 1

Structure solution and refinement.

\begin{tabular}{|c|c|c|c|c|c|c|}
\hline \multirow{2}{*}{$\begin{array}{l}\text { Condition } \\
\text { Re-refinement }(\mathrm{Y} / \mathrm{N})\end{array}$} & \multicolumn{2}{|c|}{ OMP added } & \multicolumn{2}{|l|}{ Apo form } & \multicolumn{2}{|c|}{ UMP added } \\
\hline & $\mathrm{N}$ & $\mathrm{Y}$ & $\mathrm{N}$ & $\mathrm{Y}$ & $\mathrm{N}$ & $\mathrm{Y}$ \\
\hline PDB code & 2za1 & $6 \mathrm{dsq}$ & $2 \mathrm{za} 2$ & $6 \mathrm{dsr}$ & $2 z a 3$ & $6 \mathrm{dss}$ \\
\hline Completeness (\%) & 98.9 & & 99.6 & & 99.0 & \\
\hline$\sigma$ Cutoff & 0.00 & & 0.00 & & 0.00 & \\
\hline No. of reflections, working set & 19268 & & 18297 & & 19658 & \\
\hline No. of reflections, test set & 1877 & & 1802 & & 1939 & \\
\hline \multicolumn{7}{|l|}{ No. of non-H atoms } \\
\hline Protein & 5109 & 4998 & 4964 & 4979 & 5098 & 5260 \\
\hline Ligand & 48 & 42 & 0 & 0 & 42 & 42 \\
\hline Water & 118 & 54 & 38 & 33 & 47 & 56 \\
\hline Total & 5275 & 5094 & 5002 & 5012 & 5187 & 5358 \\
\hline \multicolumn{7}{|l|}{ R.m.s. deviations } \\
\hline Bonds $(\AA)$ & 0.010 & 0.004 & 0.007 & 0.008 & 0.007 & 0.006 \\
\hline Water & 52 & 49 & 36 & 43 & 41 & 48 \\
\hline \multicolumn{7}{|l|}{ Ramachandran plot } \\
\hline Most favored (\%) & 76.7 & 97.4 & 79.5 & 96.7 & 82.99 & 96.85 \\
\hline Allowed (\%) & 91.07 & 99.84 & 94.37 & 100 & 93.58 & 99.84 \\
\hline All-atom clashscore $\dagger$ (percentile) & $50.11(12)$ & $7.6(99)$ & $37.33(40)$ & $10.22(97)$ & $32.66(40)$ & $6.46(99)$ \\
\hline Overall MolProbity score (percentile) & $3.72(10)$ & $1.53(100)$ & $3.22(23)$ & $1.74(99)$ & $3.37(24)$ & $1.55(100)$ \\
\hline
\end{tabular}

$\dagger$ Clashscore is the number of serious steric overlaps $(>0.4 \AA)$ per 1000 atoms.

dramatic improvements to problematic structures in an automated fashion (Joosten et al., 2014). However, in the case of these three structures, $P D B-R E D O$ was unable to improve the models to current standards of practice. Here, we describe the re-refinement of PDB entries 2za1, 2za2 and 2za3, resulting in a dramatic improvement of the models as suggested by the geometric parameters, density-fit analysis and $R$ factors (Table 1). Our most consequential finding is that re-refinement of $2 \mathrm{za} 1$ shows that the bound ligand is actually the product UMP, rather than the substrate OMP as reported (Tokuoka et al., 2008). These re-refined structures have been deposited in the PDB as entries 6dsq, 6dsr and 6dss, respectively.

\section{Materials and methods}

\subsection{Structure solution and refinement}

All three structures were re-refined from the deposited structure factors using the PHENIX suite (Adams et al., 2010). A starting model of the apoenzyme PfOMPDC (PDB entry 3n3m; Y. Liu, L. P. Kotra \& E. F. Pai, unpublished work) was used to generate a molecular-replacement solution. In order to minimize model bias, refinement was carried out after randomization of the coordinates of the input model in all cases using the 'shake' feature in phenix.pdbtools (Adams et al., 2010) with a maximum displacement of $0.2 \AA$. Coordinate randomization was accompanied by the removal of all ligands and bound water molecules to produce OMIT maps of bound ligands. Structures were validated using MolProbity and, during deposition, by the wwPDB validation service (Williams et al., 2018; Chen et al., 2010). The final re-refined structure with UMP bound in the active site of PfOMPDC was refined using REFMAC and BUSTER-TNT (Murshudov et al., 2011; Blanc et al., 2004). For comparison, electron-density maps of the deposited 2za1 structure were also generated using PHENIX, REFMAC and BUSTER-TNT. In both cases, all three refinement programs gave consistent results. Figs. 1, 2(b), 2(c) and 3 were generated from the electron-density maps produced by refinement in PHENIX using UCSF Chimera (Pettersen et al., 2004).

\section{Results and discussion}

Re-assessment of the structure of the orotidine $5^{\prime}$-monophosphate decarboxylase (OMPDC) from $P$. falciparum with substrate bound has revealed that this structure is not the Michaelis complex, as reported, but rather a product complex. The structural enzymology of OMPDC has depended on highquality structures of intermediates in the active site (Richard et al., 2018; Lewis et al., 2017; Reyes et al., 2015; Vardi-Kilshtain et al., 2013; Lee \& Houk, 1997). Additionally, the structure of OMPDC from $P$. falciparum is important because of its potential as drug target (Tokuoka et al., 2008; Fujihashi et al., 2015; Cassera et al., 2011). The presence of bound substrates in the study of Tokuoka and coworkers enhances the value of these structures to structure-based drug design (Tokuoka et al., 2008). Indeed, in silico drug-design efforts based on these structures have been reported (Takashima et al., 2012; Drinkwater \& McGowan, 2014). For both of these reasons, it is important that these structures are well determined and that the ligands are correctly identified (Touw et al., 2016). 
The enzyme OMPDC is a member of the $(\beta / \alpha)_{8}$-barrel superfamily. The $P$. falciparum OMPDC structures reported by Tokuoka and coworkers maintain this fold (Fig. 1).

However, the three structures of the set are not well validated overall (Wlodawer et al., 2018; Dauter et al., 2014;

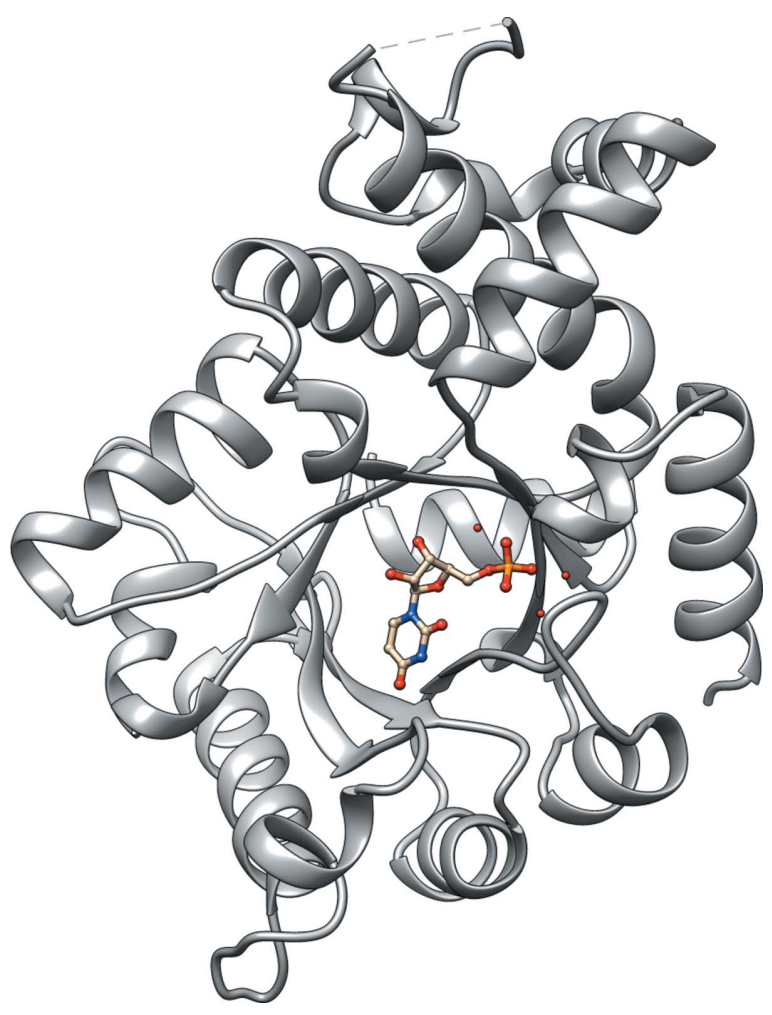

Figure 1

Schematic of the structure of the PfOMPDC monomer with the reaction product uridine $5^{\prime}$-monophosphate (UMP) bound.
Kleywegt \& Jones, 1996). By both clashscore and number of Ramachandran outliers, the OMP-bound structure with PDB code 2za1, in particular, is seen to be among the lowest scoring in the PDB (12th percentile). The re-refinement reported here solves these overall problems (Table 1, Fig. 2a). The number of residues in disfavored regions of the Ramachandran plot is reduced to an appropriate level, and the overall clashscores improve to the very topmost percentile of structures in the PDB (Fig. 2a). A specific example of the results of these improvements can be seen in Fig. 2(b), where a region of the protein that was formerly fitted as an extended loop because of a residue in a disallowed conformation is shown to form a proper $\alpha$-helix once the residue is modeled with allowed Ramachandran angles. The potential importance of the refitting to the active site is shown in Fig. 2(c), which depicts the backbone atoms in the apoenzyme that are within $5 \AA$ of where the substrate would bind. Although the original and refitted structures agree overall, regions of difference that affect the shape of the substrate-binding pocket can be observed.

More so than the global structure, modeling of the ligand in these structures is, of course, critical to their interpretation. From an enzymological perspective, the decarboxylation reaction catalyzed by OMPDC is a very well studied example of the rate acceleration that can arise from control of the electrostatic environment around a substrate (Fried \& Boxer, 2017; Amyes et al., 2017; Jordan \& Patel, 2013). OMPDC has been shown to convert substrate to product with minimal active-site rearrangement (Richard et al., 2018; Fried \& Boxer, 2017). This aspect of the enzyme is important because the experimental method of Tokuoka and coworkers for forming the co-complex of the enzyme and substrate relied on soaking the crystals in OMP. The turnover rate of the wild-type

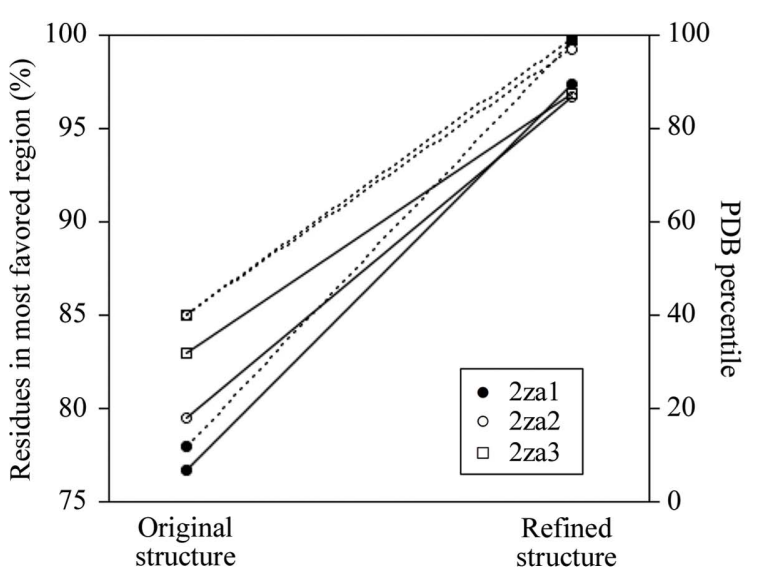

Figure 2

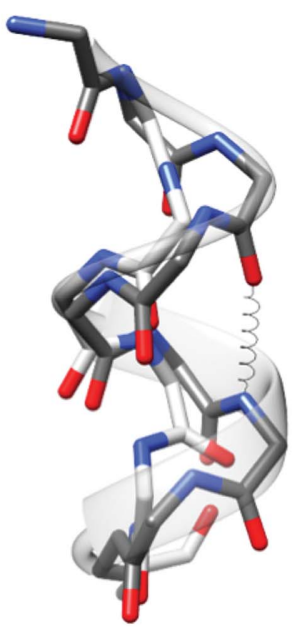

(b)

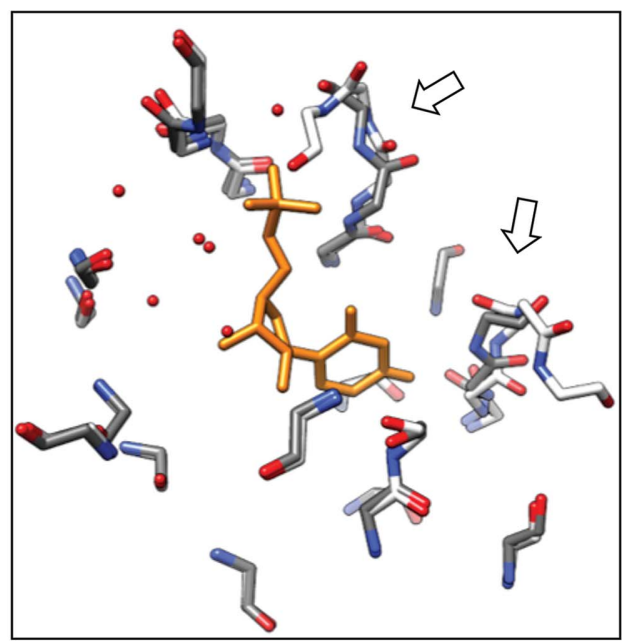

(c)

Global improvement of the PfOMPDC structure upon refitting. (a) The percentage of residues in the most favored region of Ramachandran space (solid lines) is increased in all cases. The all-atom clashscore (dotted lines), as a percentile score among all structures in the PDB, is dramatically increased. (b) An apoenzyme region originally modeled in PDB entry $2 \mathrm{za} 2$ (white) as an extended loop, owing to a residue with the backbone carbonyl misaligned, is shown by the re-refined structure (gray) to form a typical $\alpha$-helix with backbone atoms positioned within hydrogen-bonding distance (shown by the gray spring). (c) Comparison of the protein backbone near the active site shows potentially important differences (arrows) that change the shape of the binding pocket. Residues in PDB entry $2 \mathrm{za} 2$ (white) that would be within $5 \AA$ of the substrate (orange, modeled from PDB entry $2 \mathrm{za} 1$ ) are compared with those of the re-refined apoenzyme structure (gray). 


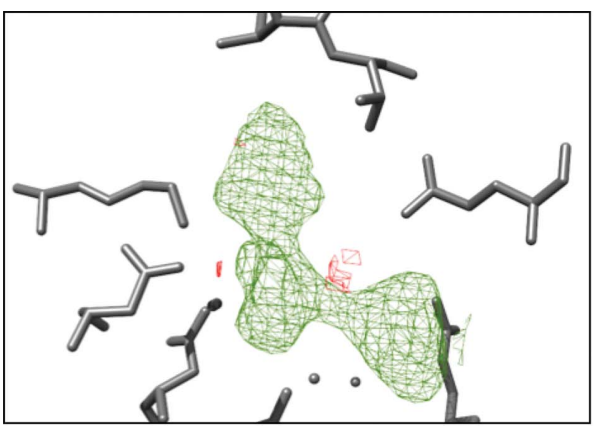

(a)

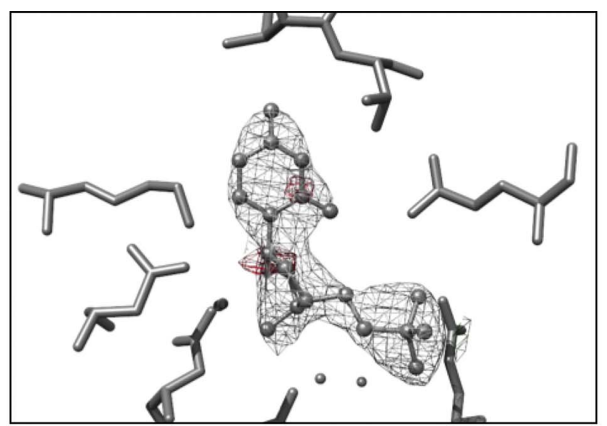

(b)

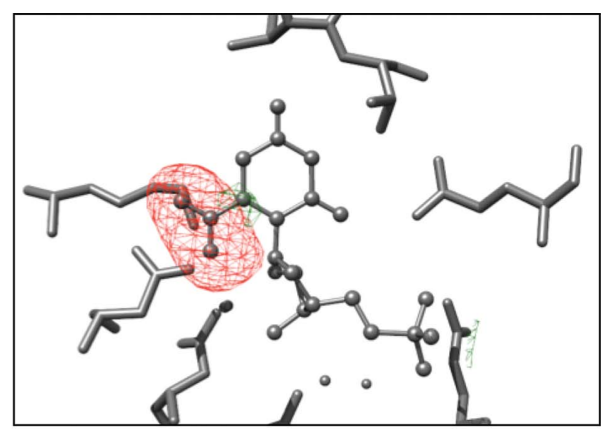

(c)

Figure 3

Ligand electron density in the PfOMPDC active site after re-refinement of the deposited structure factors (PDB entry 2za1). (a) A simulated-annealing OMIT map showing the $F_{\mathrm{o}}-F_{\mathrm{c}}$ difference density map in green for positive and red for negative difference density (both contoured at 3.0 r.m.s.d.). (b) Modeling UMP into this unbiased ligand density, showing the $2 F_{\mathrm{o}}-F_{\mathrm{c}}$ map in gray ( 1.5 r.m.s.d.) and difference density in green and red ( \pm 3.0 r.m.s.d.). (c) Modeling OMP into ligand electron density, with the difference density $F_{\mathrm{o}}-F_{\mathrm{c}}$ map in green and red ( \pm 3.0 r.m.s.d.).

enzyme is very high, and catalysis is thought to occur with little rearrangement of the active site (Fujihashi et al., 2013; Wu et al., 2000). Little barrier exists to the enzyme simply converting the substrate in these soaking experiments to product. Indeed, the originally deposited 2za1 structure itself gives evidence that the substrate OMP is an imperfect fit to the observed electron density. The $B$ factors of the carboxylate atoms are significantly higher than those of the uridine, particularly in the $B$ subunit (average carboxylate $B$ factor of $109 \AA^{2}$ compared with $73 \AA^{2}$ for the rest of the OMP cofactor). Another noteworthy aspect of modeling the carboxylate into this electron density is that the interatomic distances between the OMP carboxylate $\mathrm{O}$ atom and the nearest Asp136 carboxylate $\mathrm{O}$ atom are 2.3 and $2.5 \AA$ in the $A$ and $B$ subunits, respectively. This proximity was interpreted as evidence of the mechanistic role of electrostatic destabilization of the substrate carboxylate on the part of the enzyme (Tokuoka et al., 2008). A more parsimonious explanation, perhaps, is that the substrate carboxylate is not present in this space.

The validation metrics of the original structures raise the possibility that model bias has not been well controlled in these structures (Hodel et al., 1992). In fact, the relatively large gap observed between $R_{\text {cryst }}$ and $R_{\text {free }}$ for all three structures is a sign of overfitting (Kleywegt \& Jones, 1997). Model bias is a special concern when it comes to ligand fitting (Wlodawer et al., 2018). Unbiased re-refinement of PDB entry 2za1 shows that the OMIT map for the bound ligand contains very little electron density in the region of the carboxylate (Fig. 3a). The placement of UMP into this electron density and subsequent refinement confirms that no positive difference density exists in the carboxylate region (Fig. $3 b$ ). Finally, attempting to fit OMP into this electron density reveals the extent of negative difference density around these atoms (Fig. $3 c$ ). To be sure that the observed differences in electron density around the orotidine carboxylate were not owing to idiosyncrasies of the refinement software, both the originally deposited structure 2za1 and the re-refined structure 6dsq were evaluated using REFMAC and BUSTER-TNT to complement the PHENIX refinement. All three programs revealed pronounced negative difference density around the carboxylate in PDB entry 2za1, suggesting its absence. At the same time, all three refinement packages showed that UMP fits well into the observed electron density, without any positive difference density, in the absence of the carboxylate. Therefore, we conclude that it is the reaction product UMP and not the substrate OMP that is bound in PDB entry 2za1.

Indeed, this conclusion is consistent with the thorough reporting of Tokuoka and coworkers of the differences among the three structures, in which the two ligand-bound structures $2 z a 1$ and $2 z a 3$ are seen to be essentially identical, differing in the same ways from the apo structure $2 \mathrm{za} 2$.

\section{Funding information}

We acknowledge the support of Wabash College and the Haines Biochemistry Fund (WRPN and KHJW), and Franklin and Marshall College's Leser and Hackman Funds (GSB and LMDK).

\section{References}

Adams, P. D. et al. (2010). Acta Cryst. D66, 213-221.

Amyes, T. L., Malabanan, M. M., Zhai, X., Reyes, A. C. \& Richard, J. P. (2017). Protein Eng. Des. Sel. 30, 157-165.

Blanc, E., Roversi, P., Vonrhein, C., Flensburg, C., Lea, S. M. \& Bricogne, G. (2004). Acta Cryst. D60, 2210-2221.

Cassera, M. B., Zhang, Y., Hazleton, K. Z. \& Schramm, V. L. (2011). Curr. Top. Med. Chem. 11, 2103-2115.

Chen, V. B., Arendall, W. B., Headd, J. J., Keedy, D. A., Immormino, R. M., Kapral, G. J., Murray, L. W., Richardson, J. S. \& Richardson, D. C. (2010). Acta Cryst. D66, 12-21.

Dauter, Z., Wlodawer, A., Minor, W., Jaskolski, M. \& Rupp, B. (2014). IUCrJ, 1, 179-193.

Drinkwater, N. \& McGowan, S. (2014). Biochem. J. 461, 349-369.

Fried, S. D. \& Boxer, S. G. (2017). Annu. Rev. Biochem. 86, 387-415. Fujihashi, M., Ishida, T., Kuroda, S., Kotra, L. P., Pai, E. F. \& Miki, K. (2013). J. Am. Chem. Soc. 135, 17432-17443.

Fujihashi, M., Mnpotra, J. S., Mishra, R. K., Pai, E. F. \& Kotra, L. P. (2015). J. Genet. Genomics, 42, 221-234.

Hodel, A., Kim, S.-H. \& Brünger, A. T. (1992). Acta Cryst. A48, 851858.

Joosten, R. P., Long, F., Murshudov, G. N. \& Perrakis, A. (2014). IUCrJ, 1, 213-220.

Jordan, F. \& Patel, H. (2013). ACS Catal. 3, 1601-1617.

Kleywegt, G. J. \& Jones, T. A. (1996). Structure, 4, 1395-1400.

Kleywegt, G. J. \& Jones, T. A. (1997). Methods Enzymol. 277, 208230. 
Lee, J. K. \& Houk, K. N. (1997). Science, 276, 942-945.

Lewis, C. A., Shen, L., Yang, W. \& Wolfenden, R. (2017). Biochemistry, 56, 1498-1503.

Murshudov, G. N., Skubák, P., Lebedev, A. A., Pannu, N. S., Steiner, R. A., Nicholls, R. A., Winn, M. D., Long, F. \& Vagin, A. A. (2011). Acta Cryst. D67, 355-367.

Petsko, G. A. \& Ringe, D. (2004). Protein Structure and Function. London: New Science Press.

Pettersen, E. F., Goddard, T. D., Huang, C. C., Couch, G. S., Greenblatt, D. M., Meng, E. C. \& Ferrin, T. E. (2004). J. Comput. Chem. 25, 1605-1612.

Rathod, P. K. \& Reyes, P. (1983). J. Biol. Chem. 258, 2852-2855.

Reyes, A. C., Zhai, X., Morgan, K. T., Reinhardt, C. J., Amyes, T. L. \& Richard, J. P. (2015). J. Am. Chem. Soc. 137, 1372-1382.

Richard, J. P., Amyes, T. L. \& Reyes, A. C. (2018). Acc. Chem. Res. 51, 960-969.
Takashima, Y., Mizohata, E., Krungkrai, S. R., Fukunishi, Y., Kinoshita, T., Sakata, T., Matsumura, H., Krungkrai, J., Horii, T. \& Inoue, T. (2012). J. Biochem. 152, 133-138.

Tokuoka, K., Kusakari, Y., Krungkrai, S. R., Matsumura, H., Kai, Y., Krungkrai, J., Horii, T. \& Inoue, T. (2008). J. Biochem. 143, 6978.

Touw, W. G., Joosten, R. P. \& Vriend, G. (2016). J. Mol. Biol. 428, 1375-1393.

Vardi-Kilshtain, A., Doron, D. \& Major, D. T. (2013). Biochemistry, 52, 4382-4390.

Williams, C. J. et al. (2018). Protein Sci. 27, 293-315.

Wlodawer, A., Dauter, Z., Porebski, P. J., Minor, W., Stanfield, R., Jaskolski, M., Pozharski, E., Weichenberger, C. X. \& Rupp, B. (2018). FEBS J. 285, 444-466.

Wu, N., Mo, Y., Gao, J. \& Pai, E. F. (2000). Proc. Natl Acad. Sci. USA, 97, 2017-2022. 